\title{
Tuberculous dactylitis pseudotumor of an adult thumb: a case report
}

\author{
Freih Odeh Abu Hassan
}

Received: 23 November 2008/ Accepted: 20 January 2010/Published online: 12 February 2010

(C) Springer-Verlag 2010

\begin{abstract}
Tuberculous dactylitis is an uncommon condition which is particularly difficult to differentiate from other lesions, particularly tumors. We report the case of a 56-year-old, healthy, left-handed person who consulted for progressive painful swelling of 8-month duration in the right thumb, which had developed after direct trauma. The plain radiograph of the thumb revealed extensive destruction of the proximal phalanx associated with pathological fracture. Magnetic resonance imaging (MRI) showed replacement of the affected phalanx with prominent soft tissue mass with extension outside the bone margins. The diagnosis of tuberculous dactylitis was based on histological characteristics and positive acid fast bacilli using Ziehl-Neelsen stain. Surgical debridement and anti-tuberculous chemotherapy eradicated the infection. Seven years post treatment, the patient had good function of the thumb with no significant disability interfering with his daily activity.
\end{abstract}

Keywords Dactylitis - Tuberculosis · Granuloma · Pseudotumor

\section{Introduction}

It is of historic interest that Feilchenfeld in 1896 described tuberculous dactylitis roentgenographically in children, and

F. O. A. Hassan ( $\square)$

The Department of Orthopaedic Surgery,

Jordan University Hospital, PO Box 73, Jubaiha,

Amman 11941, Jordan

e-mail: freih@ju.edu.jo
Rankin in 1886 identified tuberculous dactylitis by histological technique [1].

Tuberculous involvement of the small bones of the hand is a rare presentation of extra pulmonary tuberculosis [2-4].

The spine is the most frequent site of skeletal involvement; occurring in less than $3 \%$ of patients with extra pulmonary tuberculosis $[5,6]$.

The many non-specific manifestations of extra pulmonary tuberculosis can cause a considerable diagnostic delay, which may be further extended, because tuberculosis as a whole has become rare and is thus easily overlooked $[3,4]$.

Tuberculous dactylitis is often referred to as spina ventosa, which is a descriptive term referring to any bone lesion that causes progressive absorption of cortex bordering the medullary canal with progressive subperiosteal hyperplasia until roentgenographically the bone appears inflated and destructed [1,7].

We describe a healthy adult patient who had tuberculous dactylitis of the proximal phalanx of the thumb and tuberculous axillary lymphadenitis associated with pathological fracture.

\section{Case report}

A 56-year-old healthy male patient presented to the outpatient department with a history of painful swelling of 8-month duration in the right thumb.

He gave a history of direct trauma to the right thumb few weeks prior the onset of the swelling, but he denied any history of contact with tuberculosis patients or pulmonary symptoms and had no treatment with antitubercular agents. 
Physical examination revealed concentric swelling of the right thumb measuring $4 \times 3 \times 3 \mathrm{~cm}$, and deformity of thumb, which was slightly tender on palpation. There was restricted movement at all joints of the thumb. Prominent enlargement of the right axillary lymph nodes was also demonstrated. Blood tests showed normal erythrocyte sedimentation rate $(15 \mathrm{~mm} 1 \mathrm{st} \mathrm{h})$ and normal white cell count $\left(4.3 \times 10^{3} / \mathrm{mm}^{3}\right)$. Radiographs of the thumb showed soft tissue swelling, extensive destruction of the proximal phalanx with subluxation of interphalangeal joint and pathological fracture (Fig. 1). A differential diagnosis of tumor or an infection was considered. A 99mTc HDP bone scan revealed increased uptake at all three phases at the joints of the right thumb and proximal phalanx. Magnetic resonance imaging (MRI) showed on T2weighted MR images, expansion of the marrow cavity of the affected phalanx, filled with heterogeneous signal intensity, consisting of a peripheral portion of higher signal intensity and a central portion of relatively low signal intensity (Fig. 2). The lesion extended to the soft tissue through the destructed margins of all sides of the phalanx, with hyperintensity noted in the subcutaneous tissue and around the tendons. On T1-weighted images, the same region showed intermediate to low signal intensity. Magnetic resonance imaging (MRI) of the right axilla showed multiple enlarged axillary lymph nodes (Fig. 3). Computerized axial scan of the thorax and abdomen did not show any primary focus of infection.

One week after complete investigations, an open biopsy was taken from the right thumb and the right axillary lymph nodes which did show necrotic like tissue. Microscopy revealed no evidence of malignancy, but granulomatous reaction with central caseous necrosis and Langhans type giant cells destroying bone trabeculae. The biopsy from axillary lymph nodes revealed replacement of the node architecture by multiple granulomas showing central necrosis. Acid fast bacilli identified by the Ziehl-Neelsen technique in the specimen culture were taken for histopathological examination after 4 weeks. One week after the confirmation of the diagnosis, surgical debridement of the granulomatous tissue at the distal 3/4 of the proximal phalanx was performed two weeks after the biopsy. A Kirschner wire was inserted to stabilize the phalanx for 6 weeks.

In the immediate postoperative period, the patient was started on antituberculous combination of ethambutol, isoniazid and rifampicin for 6 months. Patient was followed up weekly for the first month then monthly for 5 months then annually till the final follow-up. Seven years post treatment, the thumb appeared short and flexion (Fig. 4). Hand has good grip function with no significant disability (Fig. 5). Plain radiograph of the thumb confirmed healing of the disease with only remnant proximal part of the phalanx (Fig. 6).

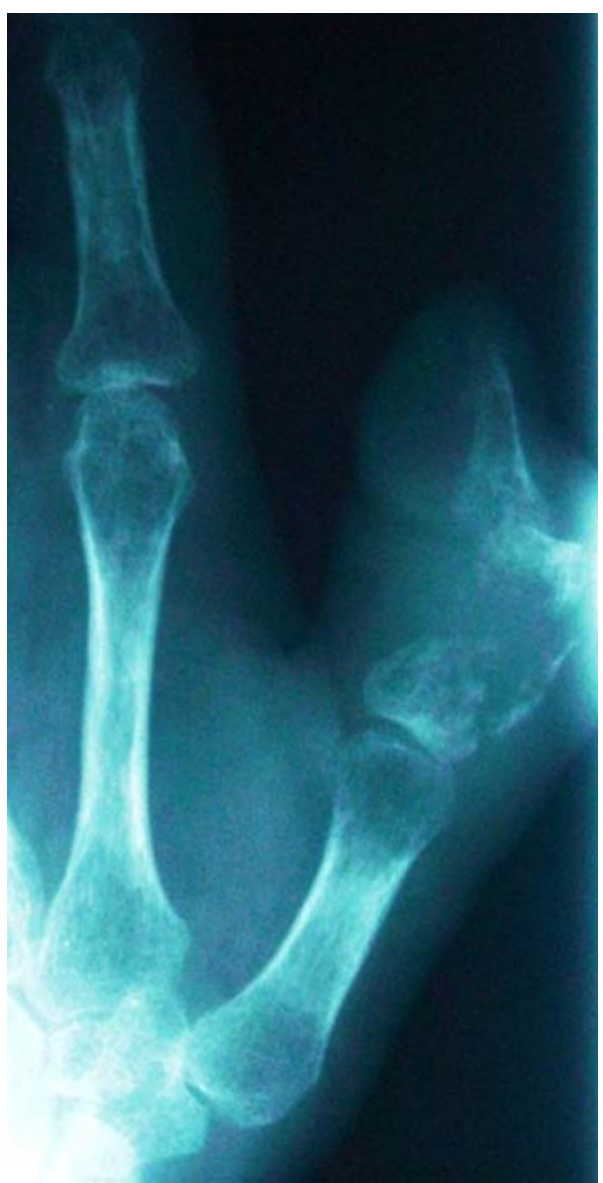

Fig. 1 Plain radiograph of the right thumb showing extensive destruction of the proximal phalanx with subluxation of interphalangeal joint and pathological fracture

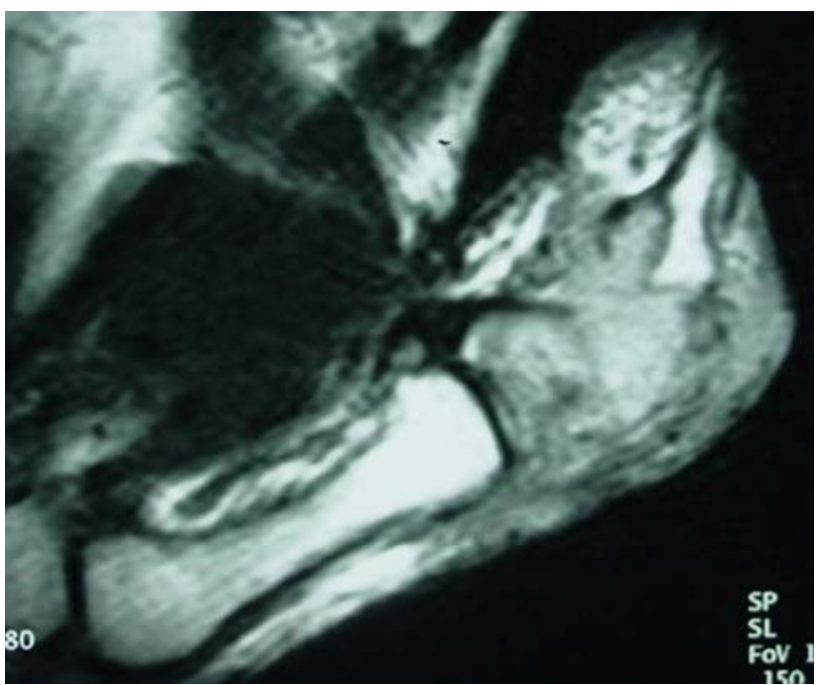

Fig. 2 T2-weighted MR images, showing expansion of the affected phalanx, filled with heterogeneous signal intensity 


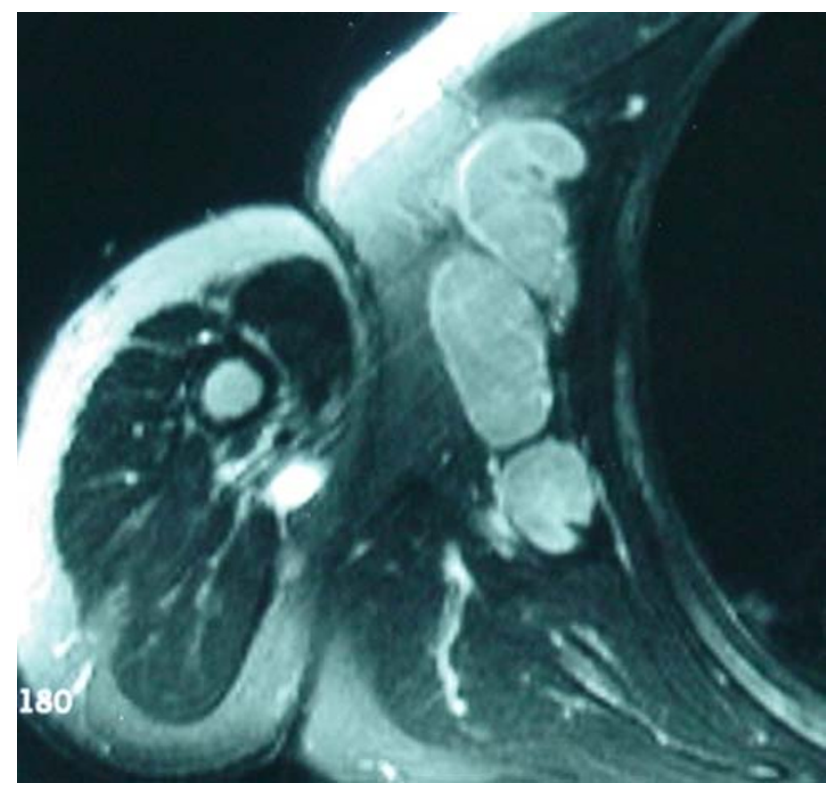

Fig. 3 Magnetic resonance imaging (MRI) of the right axilla showing multiple enlarged axillary lymph nodes

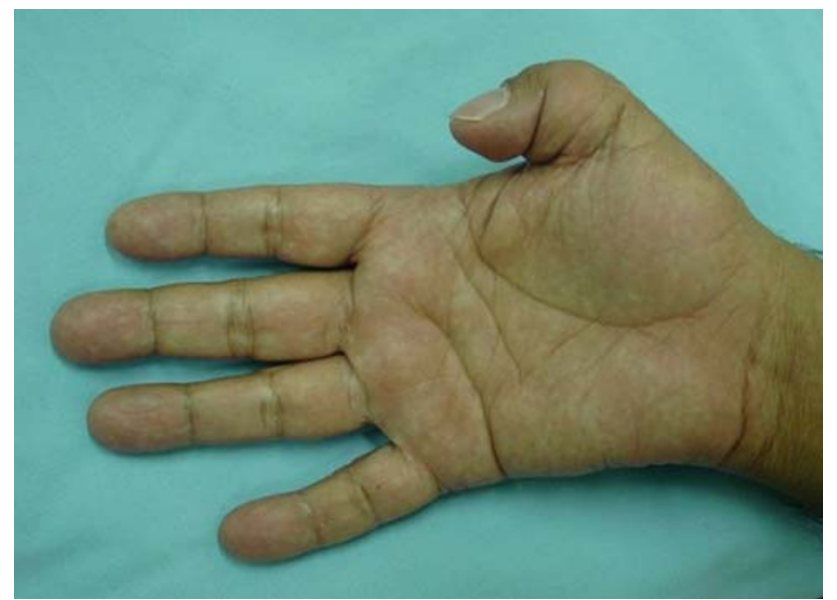

Fig. 4 Clinical appearance of the thumb at latest follow up

\section{Discussion}

Bone and joint Tuberculosis occurs in 1-3\% children who have untreated initial pulmonary tuberculosis [4, 5]. Tuberculous dactylitis runs a protracted chronic course, symptom duration ranged from a few months to 2 or 3 years after the initial infection [4, 5, 8]. Eighty-five per cent of patients with extra pulmonary tuberculosis are younger than 6 years of age, and its incidence among children with tuberculosis is reported to be $0.65-8 \%[6,7]$. Almost $50 \%$ of such cases had peripheral lymphadenopathy [9]. This was noticed in our case. In adults, extra pulmonary tuberculosis occurs between the ages of 20 and 50 $[2,3]$. The bones of the hands are more frequently affected

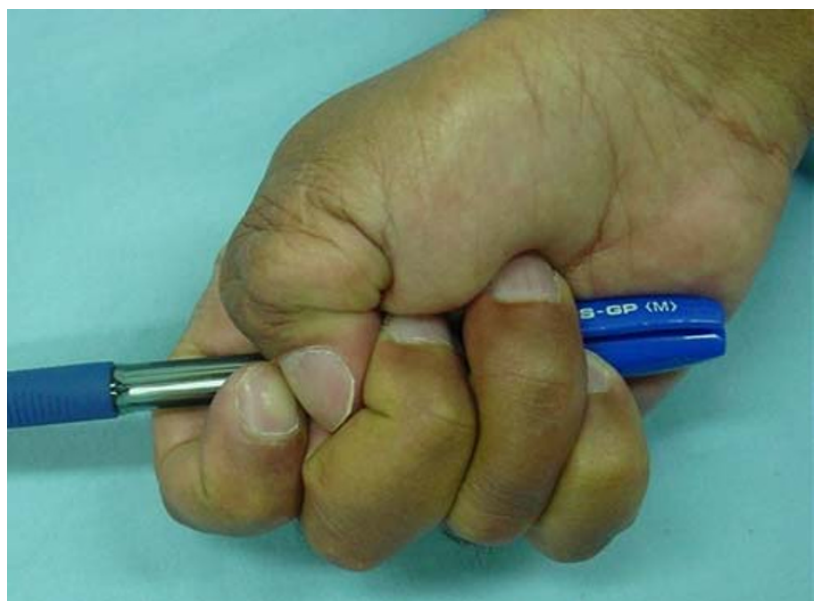

Fig. 5 Functional appearance of the hand

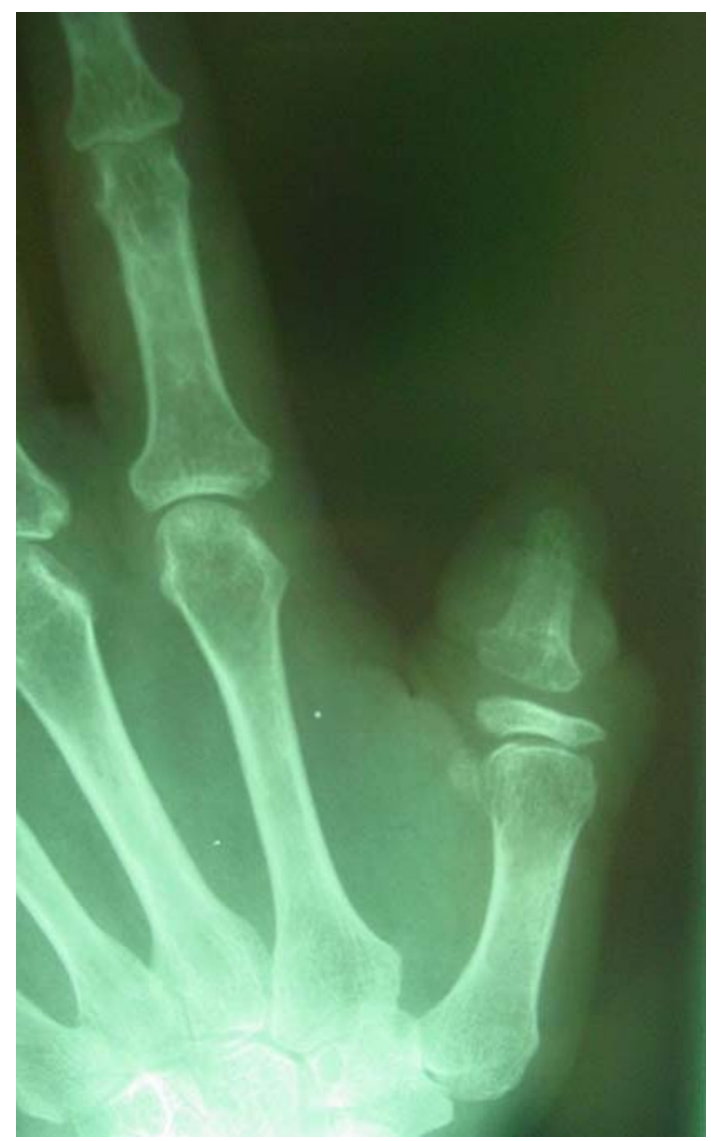

Fig. 6 Plain radiograph of the thumb confirmed healing of the disease with only remnant proximal part of the phalanx

than bones of the feet, with the proximal phalanx of the index and middle fingers the commonest sites for infection [2-4]. This is contrary to our case which occurred in the thumb. The radiographic features of cystic expansion of the short tubular bones have led to the name of "spina ventosa" being given to tuberculous dactylitis of the short 
bones of the hand [1, 7] which was noticed in our case. Periosteal reactions, sequestra and sclerosis are not common features [5, 10, 11]. Neither of these findings was noticed in our case. None of the well-known risk factors for tuberculosis e.g. socioeconomic deprivation, migration and immunodepression $[4,12]$ were noticed in our patient.

Classically, tuberculous dactylitis involves the flexor tendon sheaths and spares the joint synovium and bone in adults [13]. Our case involves both the joint and the bones with the picture of spina ventosa that peculiar to children. The definite diagnosis of tuberculous dactylitis rests on bacteriological and histological studies. The differential diagnosis of tuberculous dactylitis includes osteomyelitis, metabolic diseases (gout), sarcoidosis, and tumors [3, 14]. Infection is rapidly responsible for bone destruction, but the rate of lesion progression is far slower with mycobacteria [10]. In sarcoidosis, well-demarcated cystic lesions are found in the phalanges of the fingers, although bony expansion and periosteal new bone formation are not found $[10,14]$. In enchondromatosis, numerous lesions are present and the characteristics of punctate calcification [3, 14].

Other even more uncommon causes of lesions simulating dactylitis are hemoglobinopathies, hyperparathyroidism, and leukemia [15]. Although rare, tuberculous dactylitis must be kept in mind and needs continued vigilance when dealing with a lytic expansile lesion that involves a phalanx. Radiographic features of tuberculosis of small bones may not be recognized immediately. Despite the unusual presentation of our case in that both the joint and the small tubular bone of the thumb are nearly totally destructed with the picture of spina ventosa that peculiar to children rather than adults, eradication of infection can be performed by surgical debridement and antituberculous medications.

\section{References}

1. Pearlman HS, Warren RF (1961) Tuberculous dactylitis. Am J Surg 101:769-771

2. Leung PC (1978) Tuberculosis of the hand. The Hand 10(3):285291

3. Jensen CM, Jensen CH, Paerregaard A (1991) A diagnostic problem in tuberculous dactylitis. J Hand Surg Br 16(2):202-203

4. Subasi M, Bukte Y, Kapukaya A, Gurkan F (2004) Tuberculosis of the metacarpals and phalanges of the hand. Ann Plast Surg 53(5):469-472

5. Coombs R, Fitzgerald RH (1989) Infection in the orthopaedic patient, 1st edn. Butterworth \& Co Ltd, Great Britain, pp 297298

6. Agarwal S, Caplivski D, Bottone EJ (2005) Disseminated tuberculosis presenting with finger swelling in a patient with tuberculous osteomyelitis: a case report. Ann Clin Microbiol Antimicrob 4:18. doi:10.1186/1476-0711-4-18

7. Andronikou S, Smith B (2002) "Spina ventosa" tuberculous dactylitis. Arch Dis Child 86(3):206

8. Al-Qattan MM, Bowen V, Manteo RT (1994) Tuberculosis of the hand. J Hand Surg Br 19:234-237

9. Teo SY, Ong CL (2006) Clinics in diagnostic imaging. Singapore Med J 47(3):243-249

10. De Vuyst D, Vanhoenacker F, Gielen J, Bernaerts A, De Schepper AM (2003) Imaging features of musculoskeletal tuberculosis. Eur Radiol 13:1809-1819

11. Yoon CJ, Chung HW, Hong SH, Kim CJ, Kang HS (2001) MR findings of tuberculous dactylitis. Eur J Radiol 39(3):163-167

12. Wessels G, Hesseling PB, Beyers N (1998) Skeletal tuberculosis: dactylitis and involvement of the skull. Pediatr Radiol 28:234236

13. Albornoz MA, Mezgarzedeh M, Neumann CH, Myers AR (1998) Granulomatous tenosynovitis: a rare musculoskeletal manifestation of tuberculosis. Clin Rheumatol 17:166-169

14. Sunderamoorthy D, Gupta V, Bleetman A (2001) TB or not TB: an unusual sore finger. Emerg Med J 18(6):490-491

15. Vanmarsenille JM, de Berg B, Houssiau FA, de Selys J (2003) Unusually prolonged course of tuberculous dactylitis with osteitis. Joint Bone Spine 70:535-537 